\title{
Short-term memory for haptic cues in monkeys (Macaca mulatta)
}

\author{
RICHARD H. BAUER \\ Middle Tennessee State University, Murfreesboro, Tennessee
}

and

\author{
TERRANCE L. STEELE \\ Kansas State University, Manhattan, Kansas
}

\begin{abstract}
Short-term memory (STM) for haptic information was examined in a delayed matching-to-sample (DMTS) task. Three rhesus monkeys were tested in DMTS with all possible combinations of different-size spheres and cubes as the sample and comparison stimuli. The results showed that the decrease in percentage correct as a function of the retention intervals was relatively independent of the size and shape of the sample and comparison stimuli. In general, at each retention interval, percentage correct was higher when the (1) comparison stimuli differed in both size and shape than when the comparison stimuli differed in only size or shape, (2) size differences between the comparison stimuli increased, and (3) samples were spheres rather than cubes. This pattern of results suggests that dimensions of the haptic stimuli influenced discrimination but had little effect on the animals' STM. In Experiment 2, the same three monkeys responded to the sample and comparison stimuli with the same hand or responded to the sample with one hand and the comparison stimuli with the opposite hand. The decrease in percentage correct across retention intervals was comparable for all possible hand combinations. These results suggest that the loss of information from STM is comparable when the sample and comparison stimuli are both felt with the same hand or with opposite hands.
\end{abstract}

Short-term memory (STM) refers to the observation that a small amount of sensory information is retained for a relatively brief time (seconds) after the physical stimuli have terminated and that stored information is easily disrupted by irrelevant stimuli. Although interest in STM and other cognitive processes in animals has increased in recent years, the vast majority of animal STM experiments involve retention of visual cues. To our knowledge, only two STM studies with animals have completely excluded visual cues. Dolphins are proficient in delayed matchingto-sample (DMTS) with auditory cues (e.g., Herman, 1975), but the difficulty of maintaining dolphins in most laboratories prohibits their general use in experiments. Rats can respond when pairs of stimuli are both auditory or both visual and withhold responding when the stimulus pairs are in a different modality with delays of 0 to $5 \mathrm{sec}$ between the members of each pair (Wallace, Steinert, Scobie, \& Spear, 1980).

The STM of rats, pigeons, and monkeys has been examined in tasks with both auditory and visual cues. For ex-

We thank Susan Farber and Roger Howell for assistance in testing the monkeys and M. Marlyne Kilbey for helpful comments on the manuscript. The research was supported by a Bureau of General Research Grant.

Requests for reprints should be sent to Richard H. Bauer, Department of Psychology, Middle Tennessee State University, Murfreesboro, TN 37132. ample, rats have been trained to respond to one visual cue after presentation of an auditory sample and respond to a different visual cue after presentation of a visual sample with a few seconds delay between the auditory and visual cues (Wallace et al., 1980). Pigeons and monkeys have been trained to press a colored panel after hearing a high-frequency tone and a different colored panel after hearing a low-frequency tone with a few seconds delay between the auditory and visual cues (Dewson \& Burlingame, 1975; Kraemer \& Roberts, 1984). However, in these cross-modal STM tasks, the animals may be retaining the visual cues rather than the auditory cues.

Others have recognized the importance of developing STM tasks which require retention of nonvisual cues. However, these researchers have been unable to train monkeys in DMTS with auditory cues, in spite of extensive experience in training monkeys in DMTS with visual cues (D'Amato, 1973; Thompson, 1980). Monkeys can respond to two tones of the same frequency and withhold responding to two tones of different frequencies with a 5 -sec delay between the tone pairs (Stepien \& Cordeau, 1960); but in this study the time between the tone pairs was not varied and, for this reason, auditory STM was not investigated.

Since there is very little information concerning STM for nonvisual cues, and there is apparently no research examining STM for nonvisual cues in monkeys, an obvious extension of animal STM research is to examine 
retention curves of monkeys for nonvisual cues. Because monkeys are adept at learning various haptic discriminations (e.g., Deuel \& Mishkin, 1977; Schwartzbaum, 1965; Semmes \& Mishkin, 1965), we reasoned that monkeys could be trained to perform simultaneous matching-to-sample (SMTS) and DMTS with only haptic cues (Steele \& Bauer, 1983). The haptic SMTS task is a simultaneous discrimination task that can be turned into a STM task by inserting a delay between responding to the sample and presentation of the comparison stimuli (DMTS). In SMTS and DMTS with haptic cues, spheres or cubes of different sizes were presented on the middle of three manipulanda. The monkey felt this sample object and responded to the manipulandum. Immediately thereafter, or after a delay of a few seconds, spheres or cubes of various sizes (comparison stimuli) were presented on the two outer manipulanda (one of the objects was always the same as the sample and the other object was always different), and the monkey was reinforced for responding to the outer manipulandum which had the same shaped object as the sample.

The monkeys acquired haptic SMTS to a criterion of $90 \%$ correct in approximately 11 training days. During DMTS acquisition, percentage correct decreased from approximately $90 \%$ to $65 \%$ correct across retention intervals of .5 to $64 \mathrm{sec}$. However, the decrease across retention intervals was confounded with DMTS acquisition and, because of this confound, the decrease across retention intervals may not be an accurate representation of monkeys' STM for haptic cues. One purpose of the present studies was to examine STM for haptic information that was not confounded with acquisition. Additional purposes were to examine the effects of the physical dimensions of the haptic stimuli on STM (Experiment 1) and to compare STM when responses to the sample and comparison stimuli were made with the same hand or with the opposite hand (Experiment 2).

\section{EXPERIMENT 1}

One purpose of Experiment 1 was to provide information concerning the relationship between dimensions of the haptic stimuli and DMTS performance. Examination of the SMTS and DMTS studies with visual cues revealed that there was no consistent relationship between dimensions of visual stimuli and SMTS and DMTS performance. For example, there was no apparent relationship between the dimensions of visual stimuli and SMTS acquisition and transfer to other visual dimensions (D'Amato, Salmon, \& Colombo, 1985). Monkeys make fewer DMTS errors with visual stimuli that human observers classify as more different (Devine, Jones, Neville, \& Sakai, 1977; Jarrard \& Moise, 1971), but these results are not always consistent (Devine, Burke, \& Rohack, 1979). However, monkeys tend to make fewer matching errors when the comparison stimuli differ in both color and pattern than when they differ in only one of these dimensions (Devine et al., 1979; Devine et al., 1977), and monkeys make progressively fewer errors in haptic discriminations as differences between the haptic stimuli increase (Schwartzbaum, 1965). On the basis of the findings that monkeys make fewer matching errors when the visual stimuli differ on more than one dimension and make fewer haptic discrimination errors when the stimuli become more different, it appeared likely that performance in haptic DMTS would be higher (1) when the objects differed on two dimensions (both size and shape) than when they differed in only one dimension (size or shape) and (2) when size differences among the objects increased. At least for the objects used in the present study (spheres and cubes), there were no a priori reasons for suggesting that the shape of the objects would influence haptic matching. However, DMTS performance proved to be higher with spheres than with cubes.

\section{Method}

Subjects. Three adult male rhesus monkeys (Macaca mulatta) that had previously been trained in haptic SMTS and DMTS served as subjects (Steele \& Bauer, 1983). Throughout the experiment they were housed in individual cages and had free access to Purina Monkey Chow supplemented with fruit. On training and testing days, all fluids were obtained as reinforcement and no fluids were available in the home cage. Daily fluid consumption during this period was approximately $60 \%$ of ad-lib consumption.

Apparatus. The apparatus has been described in greater detail elsewhere (Steele \& Bauer, 1983). Briefly, a monkey was placed in a primate chair with a tube positioned in front of his mouth for delivery of $1.6 \mathrm{ml}$ grape juice reinforcements. The monkey's right hand was loosely chained, so all responses were made with the left hand. The chair was positioned in a test chamber $(60 \times 60 \times 73 \mathrm{~cm})$ so that the monkey could easily reach through a triangular hole $(15.0 \mathrm{~cm}$ wide, $8.5 \mathrm{~cm}$ from base to peak) in the chamber wall. Three manipulanda $(10.5 \mathrm{~cm}$ long, $.5 \mathrm{~cm}$ in diameter, and $5.0 \mathrm{~cm}$ apart) were attached by hinges to the outside wall immediately in front of the hole. Movement of each manipulandum by $10^{\circ}$ in any direction closed a microswitch (this defined a response) at the base of the manipulandum. The tips of the manipulanda formed an isosceles triangle with the center manipulandum $2.0 \mathrm{~cm}$ above the outer manipulanda.

Small objects $(1.5-2.5 \mathrm{~cm})$, such as knobs, hooks, and pieces of Plexiglas cut into various shapes, served as the haptic stimuli during training. During the final training phase and during testing, the stimuli were cubes and spheres with sides or diameters of 12 , 24 , or $36 \mathrm{~mm}$. These cubes and spheres were coated with polyester casting resin, to assure that each had a uniform surface. The objects were permanently attached to aluminum tubing $(9.0 \mathrm{~cm}$ long) which could be easily slipped on and off the manipulanda. The monkeys were prevented from seeing the objects by a metal plate that was attached above the hole in the chamber wall and extended to the monkey's neck. The experimenter presented the objects by rotating the manipulanda on the hinges so that the stimuli were immediately in front of the hole in the chamber wall. Retention intervals and intertrial intervals were measured by Hunter timers, which were started by responses to the manipulanda. A fan provided ventilation and masked extraneous noise.

Training procedure. The training procedure has been described in greater detail previously (Steele \& Bauer, 1983). Briefly, the monkeys were first shaped to pull each manipulandum. The monkeys were subsequently trained to discriminate between two objects placed on the outer manipulanda, and this was followed by 10-15 discrimination reversals. A criterion of 18 correct choices out of 20 consecutive trials was required during discrimination training and reversals. 
Monkeys were next trained in SMTS with the same two objects used during discrimination and reversal training. In SMTS, an object was presented on the center manipulandum (the sample stimulus) and the monkey was required to pull the center manipulandum with enough force to close the microswitch. After responding to the sample, two objects on the outer manipulanda (the comparison stimuli) were presented as rapidly as possible (within $.5 \mathrm{sec}$ ). Although simultaneous presentation of all three haptic stimuli was impossible, this procedure was called SMTS for purposes of comparison with visual DMTS studies. One of the comparison objects was always identical to the sample stimulus, and a response to this object was reinforced with grape juice. The trial terminated when a response was made to either comparison stimulus or if no response was made within $5 \mathrm{sec}$ after presentation of the comparison stimuli. To prevent the monkey from using auditory cues, all of the objects were taken off the manipulanda and placed on a cloth towel after each trial. The intertrial interval was $30 \mathrm{sec}$. The animals were required to reach a criterion of 90 correct choices out of 100 consecutive trials, followed by approximately 100 overtraining trials. A mean of 250 trials per session were given.

All monkeys were then given 13 to 27 daily sessions in which the stimuli were cubes and spheres. The monkeys were first trained on what appeared to the experimenters to be easy matches, such as large versus small spheres. This was followed either by the pairing of the small with the medium sphere and the medium with the large sphere or by the presentation of combinations of all three sizes. In the last several sessions, random presentation of all possible combinations of any two stimuli was given.

In the final training phase, a retention interval of $.5,2,4,8,16$, 32 , or $64 \mathrm{sec}$ was imposed between the response to the sample and presentation of the comparison stimuli. The monkeys received 12 daily sessions of 180 trials each. In the first four sessions, the retention intervals increased from .5 to $8 \mathrm{sec}$ and only one interval was given per session. In the next eight sessions, the three longest delays were interspersed among trials with shorter delays. Each retention interval was presented 340 times over the 12 sessions, with the exception of the 64-sec retention interval, which was presented 120 times. Percentage correct, as a function of retention intervals during the final training phase, was reported by Steele $\&$ Bauer (1983).

Testing procedure. The testing procedure was similar to that of the last training phase. The monkeys were tested in DMTS using all possible combinations of the same small, medium, and large spheres and cubes used in the last training phase. Delays of .5, 2, $4,8,16,32$, and $64 \mathrm{sec}$ were imposed between responses to the sample and presentation of the comparison stimuli. There were 24 daily sessions, each beginning with 7 warm-up trials ( 1 trial with each retention interval) followed by 175 testing trials ( 25 trials per retention interval).

In every session, each of the 15 possible pairings of comparison stimuli was presented 11-12 times. The position of the correct choice was determined at random, with the exception that the same side could be correct on no more than four consecutive trials. The order of sample presentations was determined at random, with the restriction that particular sample be presented on no more than four consecutive trials. In each session, each sample and the position of the correct choice appeared approximately an equal number of times. Across trials, the order of retention intervals was determined at random with the following restrictions: (1) that the same retention interval be presented no more than three times in succession, (2) that a maximum of four trials with short retention intervals (.5, 2,4 , or $8 \mathrm{sec}$ ) and three trials with long retention intervals (16, 32 , or $64 \mathrm{sec}$ ) be given consecutively, and (3) that each block of 20 trials contain at least one trial with each retention interval.

In the 24 sessions, there were a total of 40 trials for every possible pair of comparison stimuli at each retention interval, without regard to which of the two stimuli served as the sample and the side of the correct choice. The monkeys were run about 6 days each week throughout training and testing.

\section{Results}

Since there was heterogeneity of variance across retention intervals, the percentage of correct matches for each cell was subjected to an arcsine square-root transformation. Analyses were then done on the transformed and raw data. The conclusions based on the transformed and raw data were the same, and for this reason all analyses reported are based on the nontransformed data.

Changes across sessions. Regression analyses were used to examine changes across the 24 sessions (Cohen, 1968). Separate analyses were computed on the mean percentage correct of each monkey and for each retention interval. Regression analysis showed that for two monkeys the percentage of correct matches did not change significantly over the 24 sessions at any of the retention intervals. Across sessions, one monkey displayed a significant increase in the percentage of correct matches for four of the seven retention intervals. Collapsed over retention intervals, this represented a significant increase of $10 \%$ in correct matches $[\mathrm{F}(1,22)=24.56, \mathrm{p}<.001]$. This change across sessions in one monkey did not appear to warrant consideration in other analyses.

Retention interval and sample analyses. The mean percentage correct for each monkey, sample size, sample shape, and retention interval was determined, and a 3 (monkeys) $\times 3$ (sample size) $\times 2$ (sample shape) $\times$ 7 (retention interval) analysis of variance, with the last three factors treated as repeated measures variables, was conducted on the data. As shown in Figure 1, the percentage correct decreased as the retention interval increased $[F(6,12)=15.08, p<.001]$. (The fixed effect mean squares, i.e., the last three factors, were evaluated by the appropriate subject interaction term, and the subject effect was evaluated with the four-factor interaction.) The monkeys made significantly more correct responses when the sample stimulus was a sphere than when the sample was a cube $[F(1,2)=27.01, p<.05 ;$ see Figure 1$)$. A nonsignificant sample shape $\times$ retention interval interaction suggests that the decrease in percentage correct across retention intervals was relatively independent of the sample shapes.

Although the main effect for sample size was not significant, there was a significant monkeys $\times$ sample size $\times$ retention interval interaction $[\mathrm{F}(24,24)=3.94, \mathrm{p}<$ $.005]$. This significant interaction is apparently due to the higher matching by one monkey at longer retention intervals, especially when the sample size was large.

Comparison stimuli analyses. The effects of size and shape differences among comparison stimuli were examined by the following two methods. In the first method, trials were separated into those in which (1) shapes of the comparison stimuli were different (one stimulus was a sphere and the other a cube) but the size was the same 


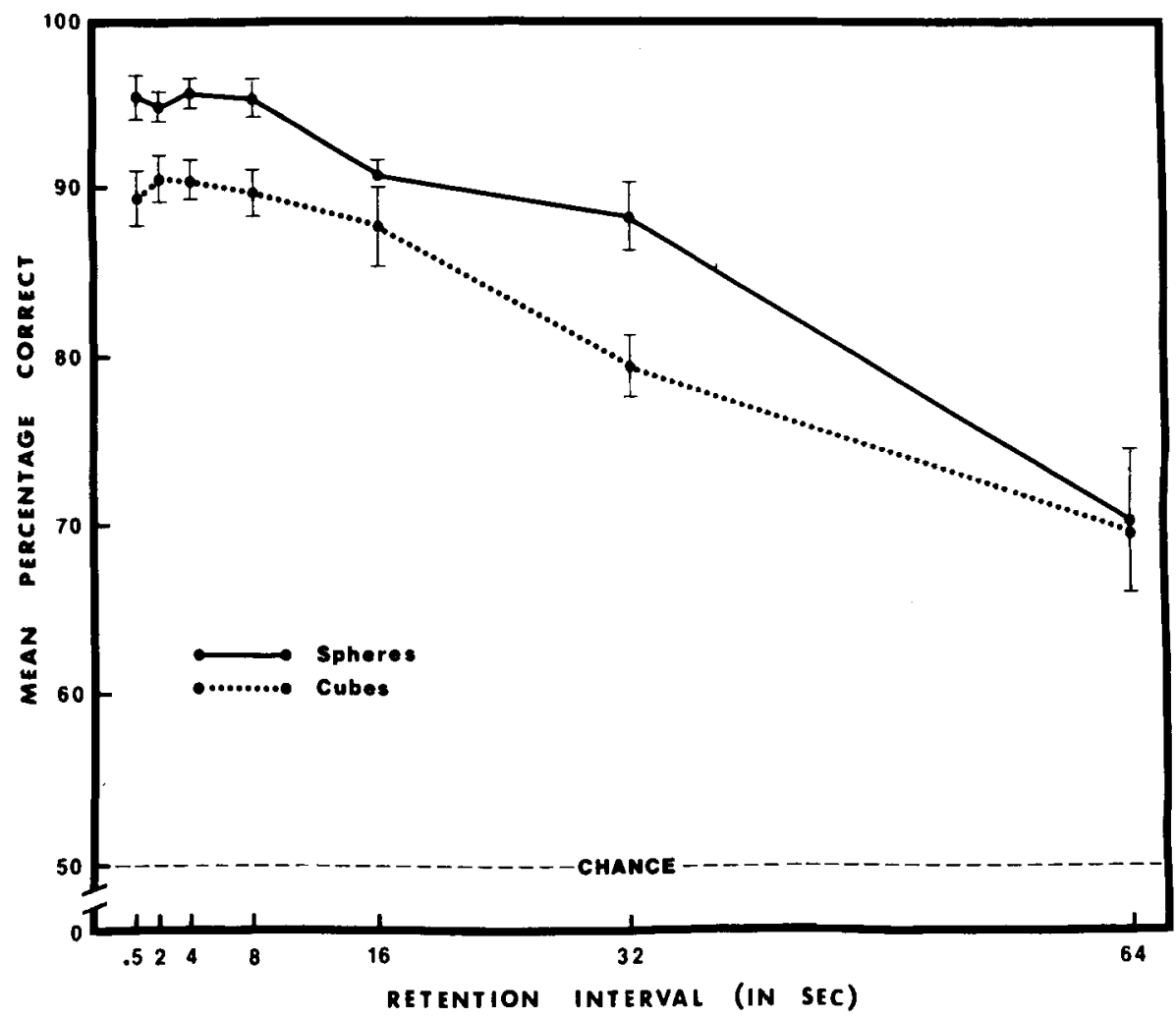

Figure 1. Mean percentage correct across retention intervals with spheres or cubes as the samples. A vertical line at each data point indicates the standard error of the mean.

(both were large, medium, or small), (2) the sizes of the comparison stimuli were different but the shape was the same (stimuli were either spheres of different sizes or cubes of different sizes), or (3) the comparison stimuli differed in both size and shape (e.g., a sphere of one size was paired with a cube of another size). In the first two trial types, the comparison stimuli differed on only one dimension (either shape or size), whereas the last combination differed on two dimensions (both shape and size). The percentage correct for each monkey, comparison stimuli combination, and retention interval was determined, and the data were analyzed by a 3 (monkeys) $\times$ 3 (comparison combination) $\times 7$ (retention intervals) analysis of variance, with the last two factors treated as repeated measures variables. The results, collapsed over monkeys, are shown in the upper left panel of Figure 2. The percentage correct decreased significantly across retention intervals $[\mathrm{F}(6,12)=18.24, \mathrm{p}<.001]$. More importantly, there was a significant main effect for comparison combinations $[F(2,4)=10.53, p<.05]$, and orthogonal comparisons revealed that matching was higher when the comparison stimuli differed on two dimensions (shape and size) than when the comparison stimuli differed on only one dimension (shape or size) $[\mathrm{F}(1,2)=34.41$, $\mathrm{p}<.05]$. There were no significant differences between comparison stimuli that differed only in size and comparison stimuli that differed only in shape. The interaction between comparison stimuli combinations and retention intervals was not significant.
Since the analysis of sample shapes revealed higher matching when the samples were spheres as opposed to cubes, the three comparison combinations (different size, different shape; different size, same shape; and different shape, same size) were further separated into trials in which the samples were either spheres or cubes. These data are presented in the upper right panel and the lower panels of Figure 2. Inspection of Figure 2 shows that, with spheres and cubes as the samples, the greatest difference occurred when the incorrect comparison stimuli differed only in size (lower left panel) and the least difference occurred when the incorrect comparison stimuli differed in both size and shape (upper right panel). A 3 (monkey) $\times$ 2 (sample shape) $\times 3$ (comparison combination) $\times 7$ (retention interval) analysis of variance (the last three factors were treated as repeated measures variables) showed significant main effects for monkeys, sample shape, comparison combination, and retention interval as well as significant interactions for subject $x$ retention interval and subject $\times$ comparison combination (all of which have been noted previously). The sample shape $x$ comparison combination interaction was not significant. However, collapsing the data across retention intervals and comparing the means by Newman-Keuls test showed that three groups of stimuli combinations were significantly different from each other. The three combinations that differ significantly, and their probability levels, are shown in Table 1.

In the second analysis method used to examine size and shape differences of the comparison stimuli, trials were 

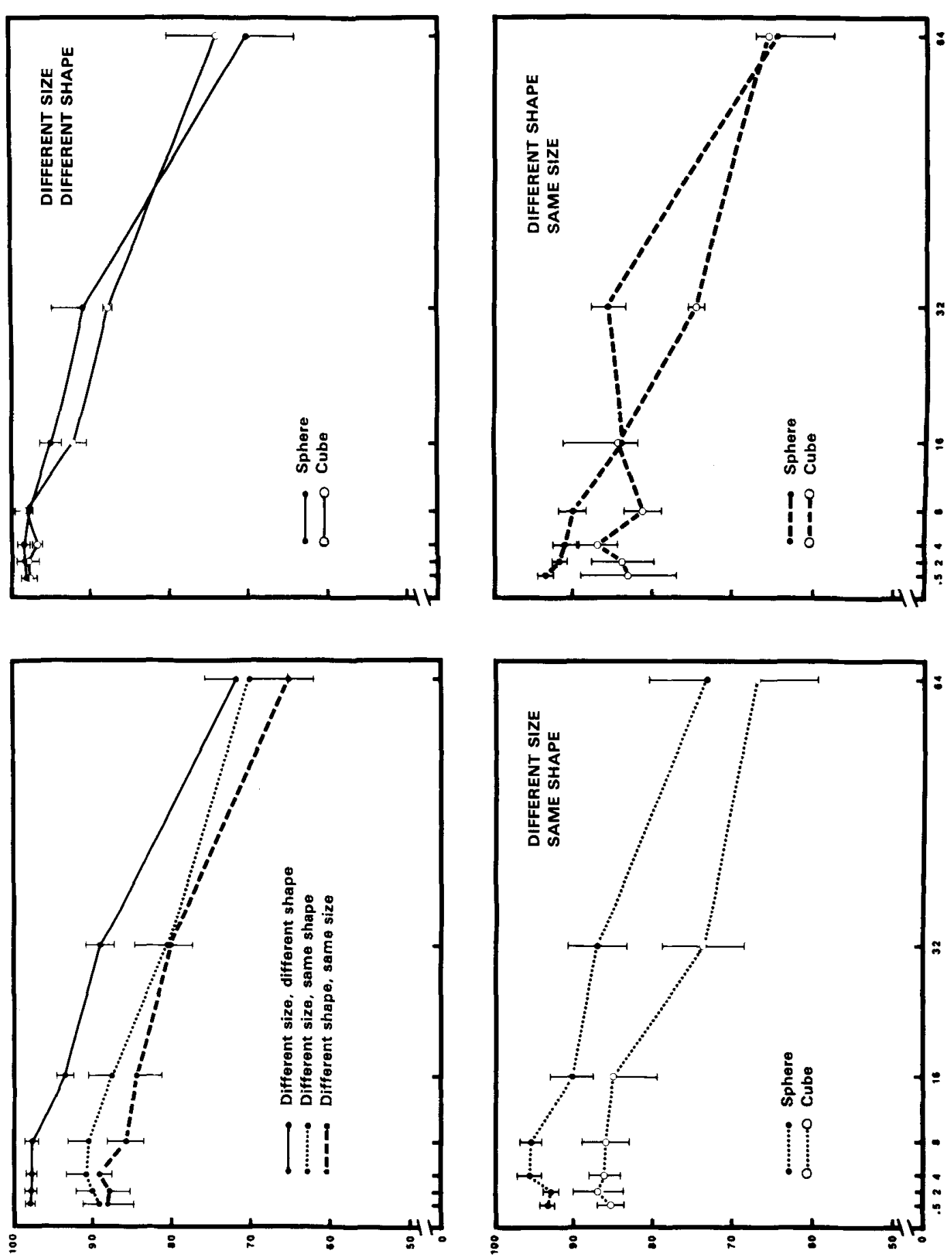

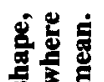

总

옹

照

要

$\frac{2}{5}$

폴

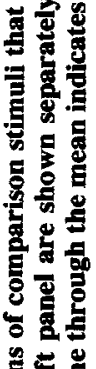

空

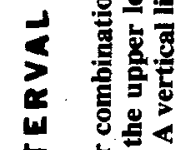

z

2 学论

2 焉

을

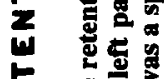

w

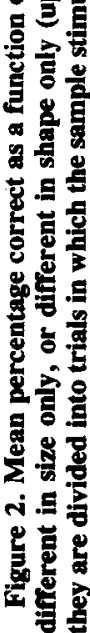

103480J 39VIN3J83d NVJW 
Table 1

Mean Percentage Correct for Samples of Different Shape and Different Combinations of Comparison Stimuli

Relationship of Comparison Stimuli

\begin{tabular}{lcccc}
$\begin{array}{c}\text { Sample } \\
\text { Shape }\end{array}$ & $\begin{array}{c}\text { Different Size } \\
\text { Different Shape }\end{array}$ & $\begin{array}{c}\text { Different Size } \\
\text { Same Shape }\end{array}$ & $\begin{array}{c}\text { Different Shape } \\
\text { Same Size }\end{array}$ & M \\
\hline Spheres & $92.45^{*}$ & $89.54 *$ & $85.71 \dagger$ & 89.23 \\
Cubes & $91.87^{*}$ & $81.34 \ddagger$ & $80.05 \ddagger$ & 84.42 \\
M & 92.16 & 85.44 & 82.88 & \\
\hline
\end{tabular}

Note-Combinations with an * are significantly higher than combinations with $\dagger(p<.05)$ and $\ddagger(p<.01)$, and $\dagger$ is significantly higher than $\ddagger(p<.05)$.

separated into those in which the diameter and length of the comparison stimuli (1) were equal (e.g., small sphere and small cube), (2) differed by $12 \mathrm{~mm}$ (e.g., small sphere and medium cube or medium sphere and large cube), and (3) differed by $24 \mathrm{~mm}$ (e.g., small sphere and large cube). For brevity, these categories are referred to as differences of 0,12 , and $24 \mathrm{~mm}$, respectively. Due to the confounding effect of shape similarity, which would occur only in the last two categories ( 2 and 3 ), comparison combinations in which the stimuli were not different in shape (e.g., small and large spheres) were not included in this analysis. Matching was highest at $24 \mathrm{~mm}$ and lowest at $0 \mathrm{~mm}$ (see Figure 3). There were significant main effects for retention interval $[\mathrm{F}(6,12)=28.01, \mathrm{p}<.001]$ and comparison combinations $[\mathrm{F}(2,6)=32.36, \mathrm{p}<.001]$. A Newman-Keuls test revealed that there were significant differences between 0 and $12 \mathrm{~mm}$ and between 0 and $24 \mathrm{~mm}(\mathrm{p}<.05)$ but no significant difference between 12 and $24 \mathrm{~mm}$.

Comparison combinations in which the diameter and length differ by $12 \mathrm{~mm}$ comprised both small and medium comparison stimuli and medium and large comparison stimuli. Since the size ratios are $1 / 2$ and $2 / 3$, respectively, matching between these two comparison combinations may differ. The percentage correct was therefore determined for small and medium combinations $(12 \mathrm{~mm} / \mathrm{small}-$ medium) and for medium and large combinations $(12 \mathrm{~mm} /$ medium-large). The percentages correct for all categories, from highest to lowest, were $93.25 \%, 92.99 \%, 90.26 \%$, and $82.85 \%$ for $24 \mathrm{~mm}, 12 \mathrm{~mm} /$ medium-large, $12 \mathrm{~mm} /$ small-medium, and $0 \mathrm{~mm}$, respectively. A Newman-Keuls test revealed that there were significant differences $(p<$ .05 ) between all means except for the difference between $24 \mathrm{~mm}$ and $12 \mathrm{~mm} /$ medium-large.

Table 2 shows the percentage correct when the samples were spheres or cubes and the comparison stimuli differed in both size and shape, differed only in size, or differed only in shape. Newman-Keuls test showed that three groups of stimulus combinations were significantly

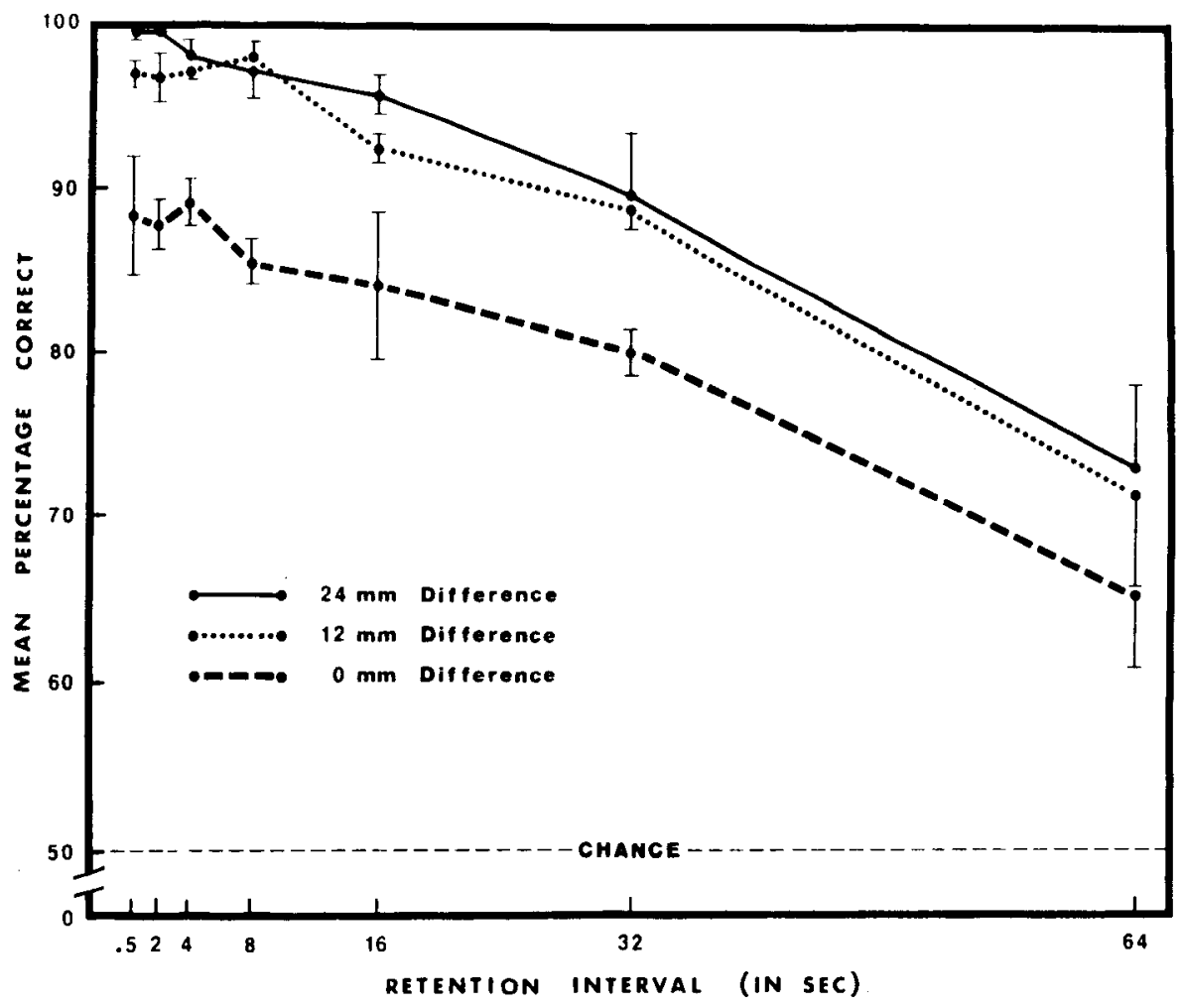

Figure 3. Mean percentage correct across retention intervals for trials in which the comparison stimuli differed in diameter or length by 0,12 , or $24 \mathrm{~mm}$. A vertical line through the mean indicates the standard error of that mean. 
Table 2

Mean Percentage Correct for Comparison Stimuli of Different Shapes and Sizes

\begin{tabular}{lcccc}
\hline & \multicolumn{4}{c}{ Comparison Stimuli Sizes } \\
\cline { 2 - 5 } Comparison & $\begin{array}{c}\text { Large and } \\
\text { Stimuli Shape }\end{array}$ & $\begin{array}{c}\text { Medium and } \\
\text { Small }\end{array}$ & $\begin{array}{c}\text { Large and } \\
\text { Sedium }\end{array}$ & M \\
\hline Spheres & $94.58^{*}$ & $91.06^{*}$ & $83.03 \dagger$ & 89.55 \\
Cubes & $90.29^{*}$ & $81.91^{\dagger}$ & $71.80 \ddagger$ & 81.33 \\
M & 92.43 & 86.49 & 77.41 & \\
\hline
\end{tabular}

Note-Combinations with an * are significantly higher than combinations with $\dagger(p<.05)$ and $\ddagger(p<.01)$, and $\dagger$ is significantly higher than $\ddagger(p<.05)$. Only combinations in which comparison stimuli were the same shape are presented.

different from each other. The three combinations that differed significantly and their associated probability levels are indicated by superscripts in Table 2 .

\section{Discussion}

The results of Experiment 1 clearly demonstrate that, with retention intervals of $2 \mathrm{sec}$ or less, monkeys perform haptic DMTS between $83 \%$ and $98 \%$ correct and that the percentage correct decreases as a function of the retention interval. Furthermore, the size and shape of the sample and comparison stimuli are important for the number of DMTS errors. In general, at each retention interval, percentage correct was higher when (1) the comparison stimuli differed in both size and shape than when they differed in either size or shape alone, (2) the size differences between the stimuli increased, and (3) the samples were spheres rather than cubes. However, the rate of decrease in percentage correct across retention intervals was relatively independent of the size and shape of the sample and comparison stimuli.

The SMTS task and the DMTS task with short delays are thought to be discrimination tasks, and errors are attributed to failure to discriminate among the relevant cues. Correct DMTS performance with longer retention intervals requires discriminating among the sensory cues, but STM becomes more important as the length of the retention interval increases. Experiment 1 showed that the dimensions of the physical stimuli influenced errors at the shortest delays, but the decrease in performance across retention intervals was independent of the physical dimensions of the relevant cues. The finding that characteristics of the relevant cues had an effect on errors at the shortest delays indicates that dimensions of the physical stimuli influenced the monkeys' discrimination. The finding that dimensions of the relevant cues had the same relative effect at both short and longer delays indicates that dimensions of the physical stimuli had little influence on the monkeys' STM.

Discriminating among haptic stimuli by monkeys is higher when the stimuli differ on a number of dimensions (Schwartzbaum, 1965), and DMTS for visual cues is higher when the stimuli differ on more than one dimension (Devine et al., 1979; Devine et al., 1977). On the basis of these findings, percentage correct was expected to be higher when the haptic stimuli differed on two di- mensions rather than just one. Matching was, in fact, higher for comparison stimuli that differed in size and shape (see Table 1 and the upper left panel of Figure 2). However, for some combinations of stimuli, haptic matching was as high when the stimuli differed on only one dimension as when the stimuli differed on two dimensions. For example, an unexpected finding was that matching was comparable (1) when both the sample and comparison stimuli were spheres and (2) when the sample was a sphere or cube and the incorrect comparison stimuli differed in both shape and size. These results are not too surprising, however, if one assumes that in some instances information for size differences alone will allow a monkey to make as many correct matches as will information on both size and shape differences. In fact, if trials are excluded in which (1) both comparison stimuli were small spheres and cubes (because of the apparent discrimination difficulty with small spheres and cubes) and (2) both the sample and comparison stimuli were cubes (because, as discussed below, size cues may not always be available for cubes), matching is roughly equivalent for the remaining combinations of comparison stimuli. Thus, although the original analyses indicated that matching was significantly higher for comparison stimuli that differed on two dimensions, as opposed to one dimension, closer inspection of the data suggests that for some combinations of comparison stimuli such a conclusion may be erroneous. These results are consistent with those of visual DMTS studies (Devine et al., 1979; Devine et al., 1977), which showed that matching on trials in which stimuli differed in both color and pattern was not always higher than matching on trials in which stimuli differed only in color or pattern.

Matching was expected to increase as the size differences between the stimuli increased. As shown in Figure 3, there was general support for this suggestion. Matching was highest when the size of the stimuli differed by $24 \mathrm{~mm}(93.25 \%)$, was slightly lower when the size of the stimuli differed by $12 \mathrm{~mm}(92.62 \%)$, and was lowest when the sizes were the same $(82.85 \%)$ ). As shown in Table 2 , for a given shape, percentage correct was highest when the size differences between the comparison stimuli were large. It should be noted that matching was higher for medium and small than for medium and large comparison stimuli, even though the size differences for these pairs of stimuli were the same. Although the size differences for medium and small and medium and large stimuli were the same, for monkeys the degree of discrimination difficulty for these pairs of stimuli are apparently not the same.

There were no a priori reasons for anticipating that DMTS errors would be influenced by the shape of the haptic stimuli. However, as shown in Figures 1 and 2, at all delays the monkeys made more correct matches when the samples were spheres than when the samples were cubes, and the decreases across the retention intervals are comparable for spheres and cubes. This pattern of results suggests that the greater matching errors with 
cubes than with spheres is due to a discrimination deficit and not to poorer retention of information for cubes. A plausible explanation for the lower discrimination of cubes is that the portion of the cube that is actually touched may not provide adequate cues for the monkeys. For example, on a given trial, a monkey may touch only a corner, portion of an edge, or portion of a flat surface. Any one of these cues would provide information that the stimulus was a cube but would not provide information concerning the size of the cube. This type of discrimination problem is less likely to occur with spheres, because the curvature of a sphere of a particular size will be the same over the entire surface and the curvatures of differentsize spheres will be different over their entire surfaces. The lower percentage correct when both the sample and comparison stimuli are cubes may be due to the fact that size cues are necessary for matching with this combination of stimuli, and that monkeys were not always provided with adequate information for the size of cubes. Furthermore, the probability of touching portions of the cubes that do not provide size cues increases as the size of the cube increases, suggesting that matching would be lower with larger than with small cubes. In support of this suggestion, more errors were committed when the sample and comparison stimuli were large and medium cubes than when they were large and small cubes or medium and small cubes (see Table 2).

\section{EXPERIMENT 2}

Sensory information from the skin, muscles, and joints of one hand is transmitted to the contralateral hemisphere, and transfer of information to the other hemisphere occurs via the corpus callosum (for a review, see Gazzaniga, 1970). In accord with these findings, humans with the corpus callosum severed make virtually no DMTS errors for haptic cues when both the sample and comparison stimuli are palpated with either the left or right hand (within hands) but are at chance when the sample is palpated with one hand and the comparison stimuli are palpated with the opposite hand (between hands) (Milner \& Taylor, 1972; Sperry, 1968). In addition, in humans, damage to one hemisphere eliminates or impairs DMTS with haptic cues when the hand used to palpate the sample and comparison stimuli is contralateral to the damage (LaMotte \& Mountcastle, 1979; Milner \& Taylor, 1972). In intact humans, however, the decrease across retention intervals from 0 to $120 \mathrm{sec}$ in haptic DMTS is comparable when the sample and comparison stimuli are both palpated with the same hand and when the sample and comparison stimuli are palpated with the opposite hands (Kleinman \& Cloninge, 1973; Milner \& Taylor, 1972). These findings with intact humans suggest that information stored in STM can be transferred between hemispheres with little or no loss of information. In intact monkeys, haptic discriminations acquired with one hand show nearly $100 \%$ transfer to the opposite hand when there are 24 or more hours between the last training session and the transfer test (e.g., Ebner \& Myers, 1962; Glickstein \& Sperry, 1960). However, STM curves for haptic cues between hands have not been reported in animals.

There are contrasting views concerning the type of information that is stored in STM. One theory suggests that stimulus-specific traces are stored (the retrospective view), whereas another view proposes that the sensory stimuli are recoded (the prospective view) (for a discussion of these contrasting views, see Kraemer \& Roberts, 1984). Since information from each hand projects to the opposite hemisphere and the cues established to the sample when responding with one hand are mirror images of the cues needed to choose the correct comparison stimuli with the opposite hand, the retrospective view predicts that withinhands matching would be higher than between-hands matching. The prospective view, however, would predict that within- and between-hands matching would be comparable. Therefore, results of the second experiment provide a clear test of these competing views of animal STM.

The major purpose of Experiment 2 was to compare STM curves of monkeys in haptic DMTS when the sample and the comparison stimuli were palpated with the same hand and when the sample and comparison stimuli were palpated with the opposite hands. Comparable within- and between-hands DMTS performance at all retention intervals would be consistent with the hypothesis that STM of haptic information is transferred between hemispheres with little loss of information and with the prospective view of STM.

\section{Method}

Subjects. The three monkeys used in Experiment 1 served as subjects.

Apparatus and Procedure. The apparatus was the same as that used in Experiment 1. The 24-mm spheres and cubes were used as sample and comparison stimuli during both training and testing.

The monkeys were trained to perform the haptic DMTS task described in Experiment 1, using only the right hand. The left hand was loosely chained, preventing the animal from reaching the stimuli. Each animal reached $90 \%$ correct in approximately 700 trials with the right hand with a delay of $.5 \mathrm{sec}$ between responding to the sample and presentation of the comparison stimuli.

The monkeys were then trained to respond to the sample with one hand and to the comparison stimuli with the opposite hand. This was accomplished by using a pulley system and two nylon cords. One end of each cord was tied to a chain on the monkey's wrists, and the other end was threaded through a small hole in the chamber wall. The experimenter prevented responding with a particular hand by pulling on the rope for that hand, making it impossible for the monkey to reach the manipulanda. During the early phases of switching between hands, the monkeys resisted having one hand held down and might not respond with the other hand. However, the monkeys gradually adapted to the procedure, and by the end of training would readily respond with the free hand. By the end of training, approximately $1 \mathrm{sec}$ was required to release one hand and to hold the other. The monkeys were given approximately 1,300 trials in which responses to the sample were made with one hand and responses to the comparison stimuli were made with the other hand. By the end of training, the monkeys were above $90 \%$ correct with all possible combinations of stimuli and hands.

The monkeys then received three daily sessions of 175 trials each, in which responses to both the sample and comparison stimuli were made with one hand and responses to the comparison stimuli were 
made with the other hand. Delays of $2,4,8,16,32$, and $64 \mathrm{sec}$ were imposed between responding to the sample and presentation of the comparison stimuli. In order to be comparable for all delays, the hands were switched immediately after the monkey responded to the sample. In each of these sessions, an equal number of trials was given with each possible combination of hands and retention intervals.

Testing. The monkeys received 16 daily sessions of 144 trials each with delays of $2,4,8,16,32$, or $64 \mathrm{sec}$ between responding to the sample and presentation of the comparison stimuli. In every session, each animal was given 6 trials with each possible combination of hands and retention intervals. The hand combinations were presented quasi-randomly with the restriction that the same hand combination be given on no more than 3 consecutive trials. The position of the correct choice, the order of sample presentations, and the order of retention intervals were as described for Experiment 1 .

\section{Results}

During both initial training with only the right hand and when switching between hands, the monkeys attempted to respond with the restrained hand, pulled on the chains on their wrists, and might not respond immediately to the sample and/or comparison stimuli. These incompatible behaviors were very likely to interfere with transfer from the left hand to the right hand and with transfer between hands. Therefore, the acquisition data obtained during training with the right hand and with switching between hands are not reported.

The number of errors for each monkey, sample shape, sample hand, comparison hand, and retention interval were converted to percentage correct. Since there was heterogeneity of variance across retention intervals, the percentage correct was subjected to an arcsine square-root transformation. A 3 (monkeys) $\times 2$ (sample shape) $\times$ 2 (sample hand) $\times 2$ (comparison hand) $\times 6$ (retention intervals) analysis of variance was computed on the transformed and nontransformed data (the last three factors were evaluated with the appropriate subject interaction terms). There were no differences in the conclusions reached on the basis of the analyses of the transformed and raw data and, therefore, the analysis of the nontransformed data are reported.

The mean percentage correct for the four possible hand combinations are presented in Figure 4 as a function of the retention interval. Matching decreased significantly across retention intervals $[\mathrm{F}(5,10)=56.65, \mathrm{p}<.001]$. There were no significant differences between responding to the sample with the left or right hand or between responding to the comparison stimuli with the right or left hand. The interaction between the sample hand and the comparison hand was also nonsignificant. In addition, the interactions involving sample hand, comparison hand, and retention intervals were not significant.

\section{Discussion}

The present study is the first to compare haptic DMTS by animals when the task is performed within hands and between hands. The results showed that the decrease in percentage correct as a function of the retention interval

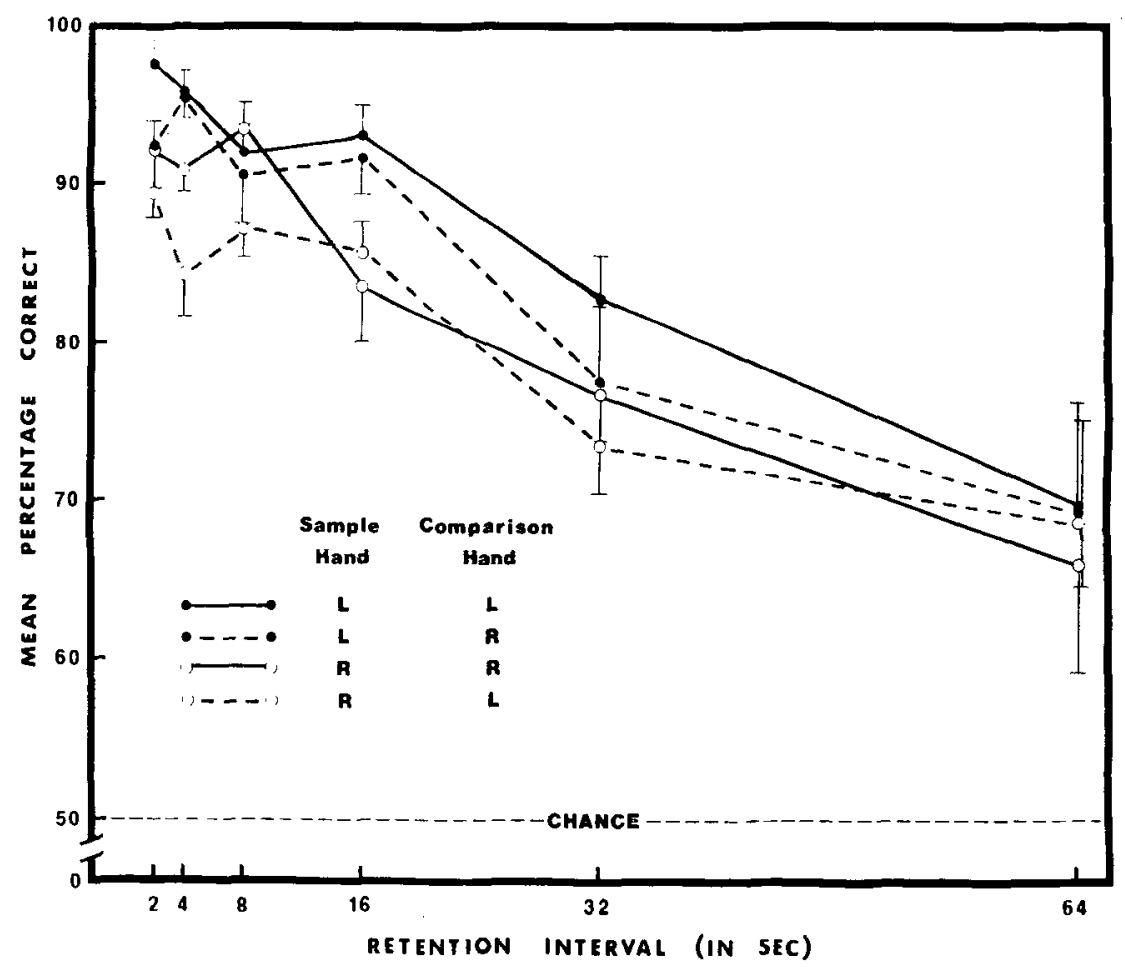

Figure 4. Mean percentage correct when the sample and comparison stimuli are both palpated with the same hand ( $L$, left; $r$, right) or with opposite hands (L-R; $R-L)$. A vertical line through the mean indicates the standard error of that mean. 
was comparable within hands and between hands. These findings with monkeys are in accord with previous reports that the decrease in haptic DMTS as a function of the retention interval by intact humans is comparable when the sample and comparison stimuli are palpated within hands and between hands (Kleinman \& Cloninge, 1973; Milner \& Taylor, 1972). These findings indicate that in both monkeys and humans STM for haptic information is readily transferred between hemispheres with little or no loss of information. The comparable within-hands and between-hands DMTS also provides clear support for the view that the relevant sensory cues are recoded (the prospective view). However, comparing the decrease in haptic matching across retention intervals indicates that the decrease is much faster in monkeys than in humans (Milewski \& Iaccino, 1982; Struss \& Butter, 1978). Therefore, the loss of haptic information stored in STM is more rapid in monkeys than in humans.

With simultaneous presentation of the sample and comparison stimuli matching-to-sample errors are thought to be due to a failure to discriminate among the relevant cues. Although it was impossible for the monkeys to simultaneously palpate all the objects, inspection of Figure 4 shows that the decrease in percentage correct across the two shortest retention intervals was negligible, suggesting that the small number of errors at short delays was due to a failure to discriminate between the sphere and cube. Since there were no significant differences among the various hand combinations used to palpate the sample and comparison stimuli, it appears that haptic discriminations can be performed at a comparable level when the information is transferred between hemispheres or remains within one hemisphere.

In some instances, intact humans perform haptic DMTS at a higher level with the left hand than with the right (Dodds, 1978; Kleinman \& Cloninge, 1973), and greater deficits in haptic DMTS are produced by damage to the right hemisphere than by damage to the left (Milner \& Taylor, 1972). On the basis of these findings, it has been suggested that, in humans, the right hemisphere plays a slightly greater role in processing haptic information than does the left hemisphere (Kleinman \& Cloninge, 1973; Milner \& Taylor, 1972). Inspection of Figure 1 shows that percentage correct by monkeys was slightly higher when only the left hand was used than when only the right hand was used. There is scattered evidence for hemispheric dominance in nonhuman primates (see the review by Denenberg, 1981), and the present results point to a right-hemispheric dominance for haptic DMTS by monkeys.

It should be noted that human and animal studies concerned with the transfer of haptic information between the hemispheres or with the effects of hemispheric damage have generally used different procedures. In human studies, some form of within- and between-hands DMTS task with retention intervals of a few seconds or minutes is commonly used (e.g., Dodds, 1978; Milner \& Taylor, 1972; Sperry, 1968). Monkey studies have typically used haptic discrimination tasks in which 24 or more hours pass between acquisition of the discrimination with one hand and the transfer test to the opposite hand (e.g., Ebner \& Myers, 1962; Glickstein \& Sperry, 1960; LaMotte \& Mountcastle, 1979). The within- and between-hands haptic DMTS procedure used in the present study is, therefore, more similar to the procedures used with humans and, for this reason, should provide a more useful animal paradigm for investigating interhemispheric transfer of haptic information.

\section{GENERAL DISCUSSION}

Comparing the decreases in percentage correct by monkeys in haptic DMTS with visual DMTS studies using similar training and test procedures indicates that the rates of decay are quite comparable (Bauer \& Fuster, 1976, 1978b; Jarrard \& Moise, 1971), but comparison of haptic DMTS with other visual DMTS studies using monkeys indicates that the rates of decay are slower or faster in haptic than visual DMTS (D'Amato, 1973; Devine et al., 1977). A variety of procedural differences have been shown to influence decay rates in visual DMTS (Bauer \& Fuster, 1978b; Cox \& D'Amato, 1982; D'Amato, 1973; Devine et al., 1977; Jarrard \& Moise, 1971), and procedural differences could be responsible for differential rates of decay in haptic and visual DMTS. Although the precise basis for differential rates of decay among DMTS studies cannot be specified, the rates of decay in haptic and visual DMTS are clearly within the same range. These findings suggest that, at least as measured by DMTS procedures, short-term retention of haptic and visual information is comparable in monkeys.

Due to the procedures used in haptic matching, the monkeys were unable to simultaneously touch all of the objects. Nevertheless, percentage correct in haptic DMTS was very similar with delays ranging from .5 to $4.0 \mathrm{sec}$, suggesting that haptic matching errors with short delays are probably due to a failure to discriminate among the objects. In addition, with delays of $2 \mathrm{sec}$ and less, the percentage correct for monkeys in haptic and visual DMTS is clearly within the same range (Bauer \& Fuster, 1976, 1978a; D'Amato, 1973; Jarrard \& Moise, 1971). These findings are consistent with previous results showing that acquisition rate and asymptotic performance for haptic and visual discriminations are comparable (Deuel \& Mishkin, 1977; Semmes \& Mishkin, 1965) and that acquisition of matching-to-sample with haptic and visual cues are also comparable (Steele \& Bauer, 1983).

In the haptic system the perception of objects is obtained by the combination of sensory information obtained from the tactile system and information obtained from proprioceptive feedback from joints and muscles (Gibson, 1966). Since the objects used in the present study were coated with a plastic resin and had a uniform surface, it appears very likely that the monkeys performed haptic SMTS and DMTS on the basis of size and shape cues and not on the basis of texture cues. 
A number of observations on how the monkeys responded in the task are worth noting. For example, during the retention interval, a monkey would invariably clasp his hand around the metal of the chamber wall immediately in front of the position where the sample had been presented, which means that he did not keep his hand in the position used when touching and responding to the sample. These observations indicate that the monkeys were not using hand shape and/or position during the retention interval as cues for correct responding. When choosing the comparison stimuli, the monkeys would reach toward one side or the other and feel an object. At least with the shortest delays, if the first comparison stimulus felt was correct the monkey responded, but when the first object felt was incorrect the animal switched to the other side. Thus, the monkeys could respond correctly without touching the incorrect comparison object. Furthermore, with the largest samples and the smallest incorrect comparison stimuli, the monkeys would reach toward a comparison object with the hand held open, much as they did when responding to the large sample. If the side with the smallest object was selected, the hand would be cupped around the smaller object, but without even touching the object the monkey would switch to the other side. Thus, with some stimulus combinations, the monkeys switched to the correct comparison object without touching the incorrect object. These observations suggest that the animals retained an internal representation of the sample stimuli and matched this internal representation with the corresponding comparison stimuli. This suggestion is in accord with the finding that neuronal activity initiated by the sample and extending into the retention interval is related to the comparison object selected by the animal. In fact, in some instances, neuronal activity during the retention interval allows one to predict the comparison stimulus the animal will select before the animal responds (Rosenkilde, Bauer, \& Fuster, 1981).

Due to our success at training and testing monkeys in haptic DMTS, an automated version of haptic DMTS has been developed. The present findings and an automated procedure should prove valuable in a variety of applications. For example, (1) Medin and Cole (1975) have indicated that there is a need to develop new procedures to increase the scope and power of STM theories. The haptic DMTS procedure will allow the generality of current theories to be tested and extended with another modality. In addition, (2) there is considerable interest in the neural mechanisms of STM (e.g., Bauer \& Fuster, 1976, 1978a; Rosenkilde et al., 1981), hemispheric dominance, and interhemispheric transfer of information (e.g., Gazzaniga, 1979). The haptic DMTS task should allow one to examine the neural bases for short-term retention of haptic information, hemispheric dominance, and interhemispheric transfer of haptic information. Also, (3) interest in the deterioration of STM with age (e.g., Bartus, Dean, \& Beer, 1980) and the effects of drugs on STM (e.g., Bartus et al., 1980; Bauer \& Fuster, 1978b) has recently increased, and the generality of age changes and drug effects can be examined and extended with haptic DMTS. Finally, (4) derived problems in cognition, perception, and sensory physiology can be examined with haptic DMTS.

\section{REFERENCES}

Bartus, R. T., Dean, R. L., \& BeER, B. (1980). Memory deficits in aged cebus monkeys and facilitation with central cholinomimetics. Neurobiology of Aging, 1, 145-152.

BaUer, R. H., \& FUSTER, J. M. (1976). Delayed-matching and delayedresponse deficit from cooling dorsolateral prefrontal cortex in monkeys. Journal of Comparative \& Physiological Psychology, 90, 293-302.

BAUER, R. H. , \& FUSTER, J. M. (1978a). The effect of ambient illumination on delayed-matching and delayed-response deficits from cooling dorsolateral prefrontal cortex. Behavioral Biology, 22, 60-66.

BAUER, R. H., \& Fuster, J. M. (1978b). Effects of d-amphetamine and prefrontal cortical cooling on delayed matching-to-sample behavior. Pharmacology Biochemistry \& Behavior, 8, 243-249.

CoHEN, J. (1968). Multiple regression as a general data-analytic system. Psychological Bulletin, 70, 426-443.

Cox, J. K., \& D'Amato, M. R. (1982). Matching to compound samples by monkeys (Cebus apella): Shared attention or generalization decrement? Journal of Experimental Psychology: Animal Behavior Processes, 8, 209-225.

D'Aмato, M. R. (1973). Delayed matching and short-term memory in monkeys. In G. H. Bower (Ed.), The psychology of learning and motivation: Advances in research and theory (Vol. 7, pp. 227-269). New York: Academic Press.

D'Amato, M. R., SAlmon, D. P., \& Colombo, M. (1985). Extent and limits of the matching concept in monkeys (Cebus apella). Journal of Experimental Psychology: Animal Behavior Processes, 11, 35-51.

DENENBERG, V. H. (1981). Hemispheric laterality in animals and the effects of early experience. Behavioral \& Brain Science, 4, 1-49.

Deuel, R., \& MishKIN, M. (1977). Limbic and prefrontal contributions to somesthetic learning in monkeys. Brain Research, 132, 521-535.

Devine, J. V., Burke, M. W., \& Rohack, J. J. (1979). Stimulus similarity and order as factors in visual short-term memory in nonhuman primates. Journal of Experimental Psychology: Animal Behavior Processes, 5, 335-354.

Devine, J. V., Jones, L. C., Neville, J. W., \& Sakai, D. J. (1977). Sample duration and type of stimuli in delayed matching-to-sample in rhesus monkeys. Animal Learning \& Behavior, 5, 57-62.

Dewson, J. H., III, \& Burlingame, A. C. (1975). Auditory discrimination and recall in monkeys. Science, 187, 267-268.

Dopds, A. G. (1978). Hemispheric differences in tactuo-spatial processing. Neuropsychologia, 16, 247-254.

EBNER, F. F., \& MYers, R. E. (1962). Direct and transcallosal induction of memories in the monkey. Science, 38, 51-52.

Gazzaniga, M. S. (1970). The bisected brain. New York: AppletonCentury-Crofts.

GIBSON, J. J. (1966). The senses considered as perceptual systems. New York: Houghton Mifflin.

GlicksteIN, M., \& SPERRY, R. W. (1960). Intermanual somesthetic transfer in split-brain monkeys. Journal of Comparative \& Physiological Psychology, 53, 322-327.

HERMAN, L. M. (1975). Interference and auditory short-term memory in the bottlenosed dolphin. Animal Learning \& Behavior, 3, 43-48.

JARRARD, L. E., \& MoISE, S. L. (1971). Short-term memory in the monkey. In L. E. Jarrard (Ed.), Cognitive processes of nonhuman primates (pp. 1-24). New York: Academic Press.

Kleinman, K. M., \& Cloninge, L. (1973). Intermanual transfer of tactual learning as a function of stimulus meaningfulness. Perceptual \& Motor Skills, 37, 875-880.

Kraemer, P. J., \& Roberts, W. A. (1984). Short-term memory for visual and auditory stimuli in pigeons. Animal Learning \& Behavior, 12, 275-284.

LaMotte, R. H., \& Mountcastle, V. B. (1979). Disorders in 
somesthesia following lesions of parietal lobe. Journal of Neurophysiology, 42, 400-419.

MediN, D. L., \& Cole, M. (1975). Comparative psychology and human cognition. In W. K. Estes (Ed.), Handbook of learning and cognitive processes: (Vol. 1). Introduction to concepts and issues (pp. 111149). Hillsdale, NJ: Erlbaum.

MilewsKi, A. E., \& IACCINo, J. (1982). Strategies in cross modality matching. Perception \& Psychophysics, 31, 273-275.

MiLnER, B., \& TAYLOR, L. (1972). Right hemisphere superiority in tactile pattern-recognition after cerebral commissurotomy: Evidence for nonverbal memory. Neuropsychologia, 10, 1-15.

Rosenkilde, C. J., Bauer, R. H., \& Fuster, J. M. (1981). Single cell activity in ventral prefrontal cortex of behaving monkeys. Brain Research, 209, 375-394.

SCHWARTZBaum, J. S. (1965). Discrimination behavior after amygdalectomy in monkeys: Visual and somesthetic learning and perceptual capacity. Journal of Comparative \& Physiological Psychology, 60, 314-319.

Semmes, J. S., \& Mishrin, M. (1965). Somatosensory loss in monkeys after ipsilateral cortical ablation. Journal of Neurophysiology, 28, 473-486.
SPERRY, R. W. (1968). Hemispheric deconnection and unity of conscious awareness. American Psychologist, 23, 723-733.

Steele, T. L., \& Bauer, R. H. (1983). A method for examining shortterm retention of haptic cues in monkeys. Behavior Research Methods \& Instrumentation, 15, 344-349.

Stepien, L. S., \& Cordeau, J. P. (1960). Memory in monkeys for compound stimuli. American Journal of Psychology, 73, 388-395.

Struss, E. H., \& BUTTER, R. B. (1978). The effect of varying types of interference on haptic memory in the Korsakoff patient. Neuropsychologia, 16, 81-90.

Thompson, R. K. R. (1980). Auditory cued reversal and matching-tosample learning by rhesus monkeys. Antropolgia Contemporanea, $\mathbf{3}$, 284.

Wallace, J., Steinert, P. A., Scobie, S. R., \& Spear, N. E. (1980). Stimulus modality and short-term memory in rats. Animal Learning \& Behavior, 8, 10-16.

(Manuscript received February 25, 1985; revision accepted for publication August 22, 1985.) 